\section{BUTTERFLY FAUNA OF MELGHAT REGION, MAHARASHTRA}

\section{Mamata Chandrakar ${ }^{1}$, Sachin Palekar ${ }^{2}$ and Sangita Chandrakar $^{3}$}

\author{
1,2 Department of Zoology, Govt. Vidarbha Institute of Science \& \\ Humanities, Amravati, Maharashtra, India \\ ${ }^{3}$ Department of Geography, Govt. Vidarbha Institute of Science \& \\ Humanities, Amravati, Maharashtra, India \\ Email: ${ }^{1}$ mamatachandra@rediffmail.com
}

plus web supplement of 3 pages

India possesses 1501 species of butterflies (Kunte et al., 1999) out of which 45 species were noted in Melghat Tiger Reserve in Melghat region, Maharashtra by Mahabal (2005). The Melghat region is noted for its rich species diversity owing to the southern tropical dry deciduous type of forest and temperature and rainfall variation. A study of butterfly diversity was conducted from April 2004 to April 2005 to compare with earlier study and to record abundance, months of abundance, and larval and adult food plants.

Melghat Region $\left(20^{\circ} 51^{\prime}-21^{\circ} 46^{\prime} \mathrm{N} \& 76^{\circ} 38^{\prime}-77^{\circ} 33^{\prime} \mathrm{E}\right)$ with a total area of $3970 \mathrm{~km}^{2}$ is given to Dharni \& Chikhaldadra tehsils in Amravati District, Maharashtra. It includes four protected areas totalling $1676.93 \mathrm{~km}^{2}$-- Chikhaldara Wildlife Sanctuary, Gugamal National Park, Melghat Tiger Reserve established in 1973, and Wan Sanctuary (Mahabal, 2005).

Melghat region experiences tropical climate with temperature ranging between $13^{\circ} \mathrm{C}$ and $22^{\circ} \mathrm{C}$ during winter and between $23^{\circ} \mathrm{C}$ and $45^{\circ} \mathrm{C}$ during summer. The annual rainfall ranges between $1000 \mathrm{~mm}$ and $2250 \mathrm{~mm}$. A total of 715 plant species are recorded here (Mahabal, 2005).

Survey collection identification: Preliminary survey was conducted to identify areas with large populations of butterflies. Ten locations were selected (Fig. 1) which were visited every month from April 2004 to April 2005. Data on butterfly fauna, its abundance, seasonality, and larval and adult food plant is based on observation from 0700-1100hr \& 1300$1700 h r$. Collection of specimens was avoided to the extent possible. Mostly photographic documentation was done (see web supplementw). When identification was not possible through photographs only then was the individual collected using net. The collected specimens were killed and preserved dry, appropriately labeled with sample number, data, locality, colour remarks etc. (Arora, 1990). The specimen collected are kept in the museum, Department of Zoology, Govt. Vidarbha Institute of Science \& Humanities, Amravati.

The butterflies were identified by using various field guides and other available literatures (Antram, 2002; Wynter -Blyth, 1957; Evans, 1932; Kunte, 2000; Sharma, 2005). Species classification and scientific names are as per Varshney (1993, 1994, 1997).

Butterflies were categorized into five groups based on their occurrence during the period of study. Accordingly, those species observed $80-100 \%$ of the survey days were categorized as very common ( $\mathrm{VC}), 60-80 \%$ as common $(\mathrm{C}), 40-60 \%$ as occasional $(\mathrm{O}), 20-40 \%$ as rare $(\mathrm{R})$, and below $20 \%$ as very rare (VR).
Results: Melghat region provides diverse habitat to various butterfly species. The study revealed the occurrence of 51 species belonging to 35 genera distributed over seven families from the monitoring sites (Table 1). The family Pieridae dominated with 14 species, followed by Nymphalidae (12 spp.), Papilionidae (9 spp.), Danaidae, Satyridae, Lyceinidae (5 spp. each) and Hesperidae (1 sp.).

Three species of butterflies recorded from this region have a protected status under the Indian Wildlife (Protection) Act, 1972 (Arora, 2003) -- Pachliopta hector Linnaeus and Hypolimnas misippus Linnaeus under Schedule I Part IV and Appias albina Boisduval under Schedule II Part II.

Further research with reference to threats and conservation of butterflies in this area is under progress.

\section{ReFERENCES}

Antram, C.B. (2002). Butterflies of India. A Mittal Publication, New Delhi, 226pp.

Arora, G.S. (1990). Lepidoptera, collections and preservation of Animals Zoological Survey of India, Kolkata, 131-137pp.

Arora, K. (2003). Forest Laws. The Wildlife Protection Act, 1972 as amended by the Wild (Protection) Amendment Act, 2002. Professional Book Publishers, New Delhi, 85pp.

Evans, W.H. (1932). Identification of Indian Butterflies. Bombay Natural History Society. Bombay, 454pp.

Kunte, K. (2000). India - A Lifescape Butterflies of Peninsular India. Indian Academy of Sciences, Bangalore, Universities Press, 270pp.

Kunte, K., A. Joglekar, G. Utkarsh \& P. Padmanabhan (1999). Patterns of butterfly, bird and tree diversity in the Western Ghats. Current Science $77(4): 577-586$.

Mahabal, A. (2005). An overview. Fauna of Melghat Tiger Reserve. Conservation Area Series, 24. Zoological Survey of India, Kolkata, 1$10 \mathrm{pp}$.

Sharma, R.M. \& C. Radhakrishnan (2005). Insecta: Lepidoptera (Rhopalocera and Grypocera) Fauna of Melghat Tiger Reserve, Conservation Area Series, 24. Zoological Survey of India, Kolkata, pp.377400 .

Varshney, R.K. (1993, 1994, \& 1997). Index Rhopalocera Indica Part III. Genera of Butterflies from India and Neighbouring countries. Oriental Insects 27: 347-372; 28: 51-198; 31: 8-137; Index. 138A-C.

Wynter-Blyth, M.A. (1957). Butterflies of The Indian Region. Bombay Natural History Society, Bombay, 523pp + 72pls.

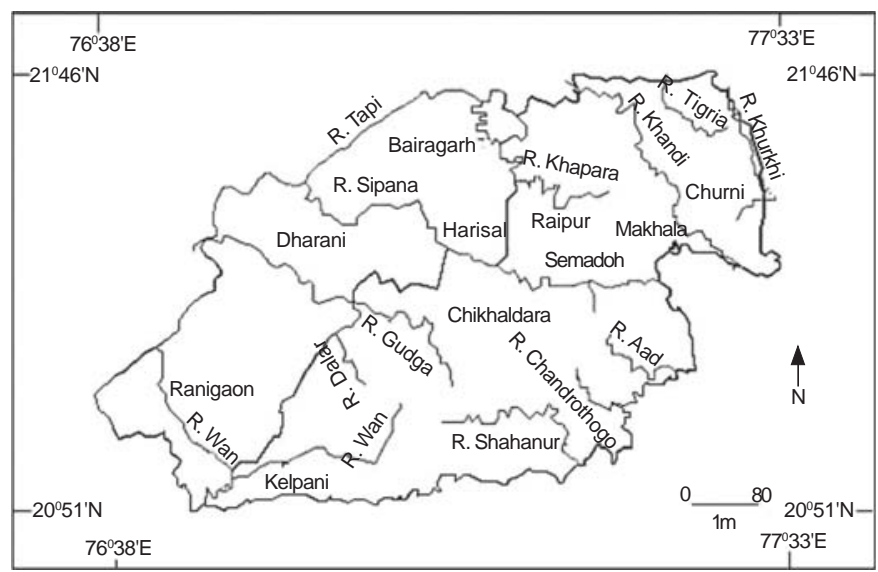

Figure 1. Map of Melghat region and sites of collection

${ }^{\mathrm{w}}$ see images in the web supplement at www.zoosprint.org

Manuscript 1479; ( ZOO; Date of publication 21 June 2007 Received 10 December 2005; Revised received 19 March 2007; Finally accepted 06 June 2007 
Table 1. Occurrence, months of abundance, larval plants and adult plants of Butterflies of Melghat region in Maharashtra

\begin{tabular}{|c|c|c|c|c|c|}
\hline & Species & Occurrence & $\begin{array}{l}\text { Months of } \\
\text { abundance }\end{array}$ & Larval plants & Adult plants \\
\hline 1 & Acraea terpiscore Linnaeus & VC & Sep-Mar & Family: Cucurbitaceae, Passiflora foetida & flower ,Tridax spp, Lantana sp. \\
\hline 2 & Appias albina Boisduval & $\mathrm{R}$ & June-July & Drypetes Spp. & flowers \\
\hline 3 & Aridne aridne Linnaeus & $\mathrm{O}$ & Apr-Oct & Ricinus communis, Tragia spp. & Branches of shrub \\
\hline 4 & Belenois aurota aurota Fabricus & $\begin{array}{l}\text { O Obs in } \\
\text { bright sun light }\end{array}$ & Apr-May & Family: Capparaceae Capparis spp. & $\begin{array}{l}\text { Flowers, Tridax procumbus, } \\
\text { Lantana camera, Zinnia elegans, } \\
\text { Impatiens balsamina, Agratum } \\
\text { conyzoides }\end{array}$ \\
\hline 5 & Catochrysops strabo Fabricus & $\mathrm{O}$ & Oct-Dec & Family: Fabaceae, Sapindaceae & \\
\hline 6 & Catopsilia pomona Fabricus & VC & July-Dec & $\begin{array}{l}\text { Cassia tora, cassia fistula, } \\
\text { Butea spp., Bauhinia spp. }\end{array}$ & $\begin{array}{l}\text { Abutilon indicum, Boerhaavia } \\
\text { diffusa, cajanus cajan, Lantana } \\
\text { spp., Tridax procumbus, Tephrosia } \\
\text { purpurea, }\end{array}$ \\
\hline 7 & Catopsilia pyranthe pyranthe Linnaeus & $\begin{array}{l}\text { VC pre post } \\
\text { monsoon abund }\end{array}$ & $\begin{array}{l}\text { Apr-Aug } \\
\text { dant }\end{array}$ & $\begin{array}{l}\text { Cassia tora, Cassia fistula, } \\
\text { Butea spp., Bauhinia spp. }\end{array}$ & $\begin{array}{l}\text { Tridax procumbus, Lantana } \\
\text { camera, Impatiens balsamina }\end{array}$ \\
\hline 8 & Cepora nerissa nerissa Fabricus & VC & July-Dec & Family: Capparaceae Capparis spp. & Flowers, Zinnia elegans \\
\hline 9 & Chilades laius Stoll & $\mathrm{C}$ & Sep-Dec & Citrus plants & Citrus plants \\
\hline 10 & Cynthia cardui Linnaeus & $\begin{array}{l}\mathrm{C} \text { in post } \\
\text { monsoon seaso }\end{array}$ & $\begin{array}{l}\text { May-July } \\
\text { on }\end{array}$ & $\begin{array}{l}\text { Debregeasia sp., Zornia gibbosa, } \\
\text { Tricolepis sp., Blumea sp., Gnaphalium } \\
\text { luteo, Gnaphalium pulvinatum }\end{array}$ & $\begin{array}{l}\text { Bushes flowers Oxalis } \\
\text { corniculata, Tridax procumbus }\end{array}$ \\
\hline 11 & Danaus chrysippus chrysippus Linnaeus & VC & Apr-Sep & Calotropis gigantea & $\begin{array}{l}\text { Lantana sp. Trichodesma indicum, } \\
\text { Tridax procumbus, }\end{array}$ \\
\hline 12 & Danaus genutia genutia Cramer & C & Apr-Sep & $\begin{array}{l}\text { Family: Asclepiadaceae } \\
\text { Cryptolepis buchanani }\end{array}$ & $\begin{array}{l}\text { Lantana camera, Tridax } \\
\text { procumbus, Clotalaria spp., } \\
\text { Celosia argentea }\end{array}$ \\
\hline 13 & Delias eucharis Drury & $\mathrm{C}$ & Aug-Oct & Family: Loranthaceae & Flowers, Lantana camera \\
\hline 14 & Euchrysops cnejus Fabricus & $\mathrm{C}$ & Sep-Dec & $\begin{array}{l}\text { Legumes pisum sativum, Acasia spp., } \\
\text { Butea monosperma }\end{array}$ & Tridax spp. \\
\hline 15 & Euploea core core Cramer & $\begin{array}{l}\text { VC } \\
\text { abundant } \\
\text { Nov- JanTh ye }\end{array}$ & $\begin{array}{l}\text { Nov-Apr } \\
\text { ear }\end{array}$ & $\begin{array}{l}\text { Ficus racemosa, Ficus benghalensis, } \\
\text { Ficus religiosa, Hemidesmus indicus, } \\
\text { Ichnocarpus frutescens, } \\
\text { Crytolepis buchananii }\end{array}$ & $\begin{array}{l}\text { Family: Euphorbiaceae Lantana } \\
\text { spp. flower, Clotalaria plants }\end{array}$ \\
\hline 16 & Graphium agamemnon menides Felder \& Felder & $\mathrm{R}$ & Aug-Oct & $\begin{array}{l}\text { Family: Annonaceae Annona } \\
\text { squamosa, Polyalthia spp., }\end{array}$ & Lantana spp \\
\hline 17 & Graphium sarpedon teredon Felder \& Felder & $\mathrm{R}$ & May-Aug & $\begin{array}{l}\text { Family: Annonaceae, } \\
\text { Lauraceae Polyalthia spp. }\end{array}$ & Lantana spp., fermenting fruits \\
\hline 18 & Hebomoia glaucippe Linnaeus & VR & Aug & Capparis spp. & \\
\hline 19 & Hypolimnas bolina Linnaeus & $\begin{array}{l}\text { VC post } \\
\text { monsoon seaso }\end{array}$ & $\begin{array}{l}\text { Aug } \\
\text { on }\end{array}$ & $\begin{array}{l}\text { Portulacea oleracea, Sida rhombifolia, } \\
\text { Elatostemma cuneatum, Laportea interrupt }\end{array}$ & $\begin{array}{l}\text { Lantana spp., Zinnia elegans, } \\
\text { ta Celosia argentea }\end{array}$ \\
\hline 20 & Hypolimnas misippus Linnaeus & $\mathrm{C}$ & Oct-Jan & $\begin{array}{l}\text { Portulaca oleracea, Abutilon indicum, } \\
\text { Abutilon pannosum, Asystasis sp., } \\
\text { Barleria cristata, Hibiscus sp. }\end{array}$ & $\begin{array}{l}\text { Herbs and shrubs Lantana spp., } \\
\text { Zinnia spp. }\end{array}$ \\
\hline 21 & Ixias marianne Cramer & $\mathrm{R}$ & Apr-June & Capparis spp. & $\begin{array}{l}\text { Flowers, Tridax procumbus, } \\
\text { Lantana camera }\end{array}$ \\
\hline 22 & Ixias pyrene sesia Fabricus & $\mathrm{R}$ & May-July & Capparis spp. & $\begin{array}{l}\text { Flowers, Tridax procumbus, } \\
\text { Lantana camera }\end{array}$ \\
\hline 23 & Junonia almana almana Linnaeus & VR & July-Oct & $\begin{array}{l}\text { Barleria prionitis, Hygrophila } \\
\text { auriculata, Acanthus sp., Osbeckia sp. }\end{array}$ & Marigold flowers \\
\hline 24 & Junonia hierta hierta Fabricus & $\mathrm{C}$ & April-March & Barleria prionitis, Hygrophila auriculata & $\begin{array}{l}\text { Marigold flowers , Lantana spp., } \\
\text { Tridax procumbus }\end{array}$ \\
\hline 25 & Junonia lemonias lemonias Linnaeus & $\begin{array}{l}\text { VC th year } \\
\text { but monsoon \& } \\
\text { post monsoon } \\
\text { highest }\end{array}$ & Apr-Mar & $\begin{array}{l}\text { Hygrophila auriculata, Nelsonia } \\
\text { canesens, Corchorus oliforius, } \\
\text { Cannabis sativa, Sida rhombifolia }\end{array}$ & $\begin{array}{l}\text { Damp patches flowers of } \\
\text { Impatiens balsamina, Agratum } \\
\text { conyzoides, Celosia argentea, } \\
\text { Tephrosia purpurea }\end{array}$ \\
\hline 26 & Junonia orithya Linnaeus & $\mathrm{C}$ & Sep-Feb & $\begin{array}{l}\text { Justicia procumbens, Lepidagathis } \\
\text { prostrata }\end{array}$ & $\begin{array}{l}\text { Herbs, Lantana spp., Tridax } \\
\text { procumbus }\end{array}$ \\
\hline 27 & Lampides boeticus Linnaeus & $\mathrm{C}$ & Oct-Nov & $\begin{array}{l}\text { Legumes pisum sativum, Acasia spp., } \\
\text { Butea monosperma }\end{array}$ & Celosia argentea \\
\hline
\end{tabular}




\begin{tabular}{|c|c|c|c|c|c|}
\hline & Species & Occurrence & $\begin{array}{l}\text { Months of } \\
\text { abundance }\end{array}$ & Larval plants & Adult plants \\
\hline 28 & Lethe confusa aurivillius Fabricus & VR & Oct-Nov & & $\begin{array}{l}\text { Fallen fruits, dry leaves, Close } \\
\text { to ground }\end{array}$ \\
\hline 29 & Melanitis leda leda Linnaeus & VC & Sep-Dec & $\begin{array}{l}\text { Oryza sativa, Sorghum durra, } \\
\text { Sorghum dochna, Sorghum deccanense }\end{array}$ & $\begin{array}{l}\text { Fallen fruits, rotting fruits } \\
\text { flowers, Agratum conyzoides }\end{array}$ \\
\hline 30 & Melanitis phedima bela Moore & $\mathrm{R}$ & Oct-Jan & $\begin{array}{l}\text { Apluda mutica, Eleusine coracana, } \\
\text { Eleusine indica, Oplismenus compositus, } \\
\text { Oryza sativa, Sorghum durra, Sorghum } \\
\text { dochna, Sorghum deccanense }\end{array}$ & Fallen fruits, rotting fruits \\
\hline 31 & Moduza procris Cramer & VR & Feb & Mussaenda spp. & flowers \\
\hline 32 & Mycalesis perseus tabitha Fabricus & VC & Oct-Nov & $\begin{array}{l}\text { Grasses, Apluda mutica, Eleusine } \\
\text { coracana, Eleusine indica, Oplismenus } \\
\text { compositus, Oryza sativa, Sorghum durra } \\
\text { Sorghum dochna, Sorghum deccanense }\end{array}$ & $\begin{array}{l}\text { Fallen fruits, rotting fruits, dung } \\
e\end{array}$ \\
\hline 33 & Neptis hylas Linnaeus & VR & Aug-Sep & $\begin{array}{l}\text { Family-Bombaceae, Fabaceae, } \\
\text { Icacinaceae, Sterculiaceae, Tiliaceae }\end{array}$ & Nector of plant, wet patch \\
\hline 34 & Pachliopta aristolochiae aristolochiae Fabricus & C & July-Sep & Aristolochia spp. & $\begin{array}{l}\text { Lantana spp., Cosmos spp, } \\
\text { Zinnia spp. }\end{array}$ \\
\hline 35 & Pachliopta hector Linnaeus & $\mathrm{O}$ & Apr-Nov & Aristolochia spp. & Lantana spp. \\
\hline 36 & Pachliopta jophon pandiyana Moore & $\mathrm{C}$ & Apr-Nov & Aristolochia spp. & Lantana spp. \\
\hline 37 & Papilio protenor protenor Cramer & VR & July & Aegle marmelos & flowers, Lantana spp. \\
\hline 38 & Papilio demoleus demoleus Linnaeus & VC & Apr-Mar & $\begin{array}{l}\text { Glycosmis spp., Aegle marmelos, } \\
\text { Murraya spp. }\end{array}$ & $\begin{array}{l}\text { flowers, Tridax procumbus, } \\
\text { Lantana camera, Tephrosia purpurea }\end{array}$ \\
\hline 39 & Papilio polytes romulus Cramer & VC & Apr-June & $\begin{array}{l}\text { Glycosmis spp., Aegle marmelos, } \\
\text { Murraya spp. }\end{array}$ & Cassia spp., Caesalpinia spp. \\
\hline 40 & Parantica aglea aglea Stoll & $\mathrm{R}$ & Sep-Nov & $\begin{array}{l}\text { Ceropegia oculata, Calotropis } \\
\text { gigantean, Cryptolepis buchanani, } \\
\text { tylophora spp. }\end{array}$ & Clotalaria spp., Lantana spp. \\
\hline 41 & Pareronia valeria Cramer & $\mathrm{C}$ & Aug-Oct & Capparis spp. & flowers \\
\hline 42 & Pathysa nomius nomius Esper & $\mathrm{R}$ & July-Sep & $\begin{array}{l}\text { Polyathia longifolia, } \\
\text { Saccopetalum tomentosum }\end{array}$ & flowers, Lantana spp. \\
\hline 43 & Precis iphita iphita Cramer & $\mathrm{R}$ & Nov-Dec & $\begin{array}{l}\text { Strobilanthes sp., Hygrophila } \\
\text { auriculata, Asteracantha sp. }\end{array}$ & $\begin{array}{l}\text { Low growing plant flower } \\
\text { Tephrosia purpurea }\end{array}$ \\
\hline 44 & Pseudozizeeria maha Kollar & $\begin{array}{l}\mathrm{C} \text { abundant } \\
\text { post monsoon }\end{array}$ & Sep-Dec & $\begin{array}{l}\text { Nelsonia sp., Oxalis corniculata, } \\
\text { Tephrosia pauciflora }\end{array}$ & Small flowers \\
\hline 45 & Telicota ancilla Mabille & $\mathrm{R}$ & Oct-Nov & Oryza sp., Saccharum sp. & \\
\hline 46 & Terias blanda silhetana Wallace & $\mathrm{C}$ & Apr-Jan & Family: Caesalpiniaceae & $\begin{array}{l}\text { Tridax procumbus, Zinnia elegans, } \\
\text { Justicia quinauangularis, } \\
\text { Tephrosia purpurea }\end{array}$ \\
\hline 47 & Terias brigitta rubella Stoll & VC & Apr-Dec & Family: Caesalpiniaceae, Cassia spp. & $\begin{array}{l}\text { Tridax procumbus, Zinnia elegans, } \\
\text { Justicia quinauangularis, } \\
\text { Tephrosia purpurea }\end{array}$ \\
\hline 48 & Terias hecabe simulata Moore & VC & Apr-July & $\begin{array}{l}\text { Family: Caesalpiniaceae, } \\
\text { Mimosaceae, Fabaceae }\end{array}$ & $\begin{array}{l}\text { Tridax procumbus, Zinnia elegans, } \\
\text { Justicia quinauangularis, } \\
\text { Tephrosia purpurea }\end{array}$ \\
\hline 49 & Terias laeta laeta Boisduval & VC & Apr-Jan & Family: Caesalpiniaceae, Fabaceae & $\begin{array}{l}\text { Tridax procumbus, Zinnia elegans, } \\
\text { Justicia quinauangularis, } \\
\text { Tephrosia purpurea }\end{array}$ \\
\hline 50 & Tirumala limniacea Cramer & $\mathrm{C}$ & Apr-June & $\begin{array}{l}\text { Asclepias curasavica, Hoya spp., } \\
\text { Tylophora indica, Waltakaka volubilis }\end{array}$ & Clotalaria spp. Lantana spp. \\
\hline 51 & Ypthima huebneri Kirby & C & Oct-Dec & Small grasses & Close to ground \\
\hline
\end{tabular}

O - Occasional; VC - Very Common; C - Common; VR - Very Rare; R - Rare

\section{Acknowledgement}

This project was sanctioned by UGC as a Minor Research Project No: F 47-60/2003. The authors are thankful to UGC for financial assistance. We are also grateful to Sri Nitin Kakodkar, Chief Conservator of Forests IFS, Melghat Tiger Reserve, Amravati, Maharashtra, for constant encouragement and sustained support. Finally, we thank Prof. R.B. Chandrakar for his guidance. 